\title{
Clinical relevance of maximal inspiratory pressure: determination in COPD exacerbation
}

This article was published in the following Dove Press journal: International Journal of Chronic Obstructive Pulmonary Disease 21 April 2010

Number of times this article has been viewed

\author{
Voicu Tudorache' \\ Cristian Oancea' \\ Ovidiu Fira Mlădinescu² \\ 'Department of Pneumology, \\ ${ }^{2}$ Department of Pathophysiology, \\ Victor Babeș University of Medicine \\ and Pharmacy, Timișoara, Romania
}

Correspondence:Voicu Tudorache Department of Pneumology, Victor Babeș University of Medicine and Pharmacy, Eftimie Murgu sq, no 2, 30004I, Timișoara, Romania Email voicu.tudorache@yahoo.com

\begin{abstract}
Muscle dysfunction represents a pathophysiological feature of chronic obstructive pulmonary disease (COPD). Muscle impairment contributes to decreased effort capacity in these patients at least in the same proportion as pulmonary function limitation. Maximal inspiratory pressure (MIP) is a reliable, noninvasive parameter for assessing the respiratory muscle capacity. The aim of the present study was to determine the role of MIP in effort capacity decrease in COPD patients. MIP was measured in $121 \mathrm{COPD}$ patients without hyperinflation ( $\mathrm{RV}<150 \%$ ) together with the following investigations: body plethysmography, body impedance analysis, dynamometry, 6-minute walking test (6MWT), determination of $\mathrm{SaO}_{2}$ and serum levels of highly sensitive C-reactive protein (hsCRP). MIP ( $\mathrm{kPa}$ ) was significantly decreased in moderate-severe stages (6.19 \pm 2.42 , COPD II; $5.35 \pm 2.49$, COPD III; $4.56 \pm 1.98$, COPD IV vs $7.90 \pm 2.61$ in controls, $P<0.001$ ), whereas the muscle force assessed by dynamometry was decreased only in advanced stages of disease $(0.47 \pm 0.12$, COPD III; $0.41 \pm 0.07$, COPD IV vs $0.71 \pm 0.16$ in controls, $P<0.001)$. The values of MIP correlated $(\mathrm{r}=0.53, P=0.0003)$ with the distance walked in 6MWT. MIP may provide additive information concerning the general profile of muscle dysfunction in COPD patients.
\end{abstract}

Keywords: COPD, MIP, exacerbation

\section{Introduction}

Chronic obstructive pulmonary disease (COPD) is today considered a disease with multiple systemic pathological components. Muscular dysfunction represents one of these components, and in COPD patients it is responsible for the decrease in effort capacity at least in the same manner as respiratory function limitation; moreover in time it exceeds the component generated by pulmonary dysfunction due to the impact of this dysfunction on effort capacity and other components (eg, quality of life). ${ }^{1,2}$

In the context of global muscular dysfunction, inspiratory muscle function is frequently affected. Inspiratory muscle dysfunction results from the interference of thoracic geometry changes with systemic inflammatory factors and/or the structural alteration of these muscles. Inspiratory muscle dysfunction apparently does not limit resting ventilatory capacity, but seems to contribute to dyspnea, decrease in effort capacity, and respiratory failure during exacerbation (E-COPD). ${ }^{3,4,5}$

Composite indexes like BODE (Body mass index [BMI], Obstruction, Dyspnea, Exercise capacity) that incorporate some parameters related to muscles (BMI 6-minute walking test $[6 \mathrm{MWT}])$, provide a global perspective regarding muscular capacity. ${ }^{6}$ 
The analysis of certain symptoms that suggest decreased respiratory muscle function, such as vital capacity decrease without any explanation, retention of $\mathrm{CO}_{2}$ without severe obstruction of respiratory airwaves, dyspnea, orthopnea, or dyspnea during activities of daily living (ADL), tachypnea, paradoxical movement of the thoracic-abdominal wall, cough and recurrent infections, difficulties in speaking, etc, is more or less specific and indirect at the same time. ${ }^{3,7}$

Decrease in inspiratory muscle function, especially of the diaphragm muscle, may explain not only the symptomatology described above, but also the fact that it represents an important prediction factor for the survival rate in COPD patients. ${ }^{6,8,9}$

This explains why more and more specific and reliable instruments and methods for the functional assessment of these muscles are searched.

One of these methods is represented by the assessment of maximal inspiratory pressure (MIP) and maximal expiratory pressure (MEP). This noninvasive method is reliable, easy to perform and well accepted by patients, and consequently easy to apply in current clinical pratice..$^{10,11}$

Although muscle wasting is currently accepted, the questions regarding the clinical relevance of MIP determination arises and it is the main goal of our research. The second goal of the study was to investigate the correlation degree of MIP values obtained from patients, with other parameters that interfere with muscle dysfunction.

\section{Material and methods}

One hundred twenty-one COPD patients in the 10th day of treatment for exacerbation were compared to the reference group consisting of 31 healthy nonsmokers individuals. The study protocol was approved by the hospital ethics committee and patients gave their written consent. The patients with stage 2-3 E-COPD according to Anthonisen's classification had mild-severe disease (stage III-IV Global Initiative for Chronic Obstructive Lung Disease [GOLD]), ${ }^{12}$ were smokers, and during their admission in the hospital received antibiotics and also systemic corticotherapy treatment for at least 10 days. In Table 1 other features of the patients are presented compared to the E-COPD group.

Exclusion criteria were represented by: myopathy, significant hyperinflation ( $R V \geq 150 \%$ ), severe comorbidities (any form of cancer, diffuse pulmonary fibrosis or extended fibrotic sequelae, heart failure, advanced ischemic heart disease, diabetes mellitus).

Patients from the COPD group were not included in a pulmonary rehabilitation program. It was affirmed that all
Table I Characteristics of patients included in the study

\begin{tabular}{lll}
\hline & $\begin{array}{l}\text { Control } \\
\text { group } \\
\text { (sedentary-healthy) }\end{array}$ & $\begin{array}{l}\text { E-COPD } \\
\text { (hospitalized } \\
\text { pts) }\end{array}$ \\
\hline Number & 31 & 121 \\
Male/Female & $26 / 5$ & $109 / 12$ \\
Age (years) & $54 \pm 9$ & $60 \pm 12$ \\
Smoking state (pack-year) & Nonsmokers & $30 \pm 7$ \\
FEV (L/s) & $2.9 \pm 0.31$ & $1.27 \pm 0.58$ \\
FEV (\% pred) & $96.76 \pm 10.44$ & $42.46 \pm 19.45$ \\
Systemic corticotherapy & Any & Yes* \\
\hline
\end{tabular}

Note: $* 32 \mathrm{mg}$ methylprednisolone/day for 10 days with decrease in maximum 2 weeks.

Abbreviation: $\mathrm{FEV}_{1}$, forced expiratory volume in one second.

patients took the medication prescribed by the pneumology specialist in ambulatory care.

The subjects followed a certain protocol for investigations:

- Complete clinical evaluation

- Determination of the muscular force of the dominant superior limb (dynamometry)

- Determination of body composition (body impedance analysis - BIA) and of body mass index (BMI)

- Spirometry and body plethismography

- Determination of MIP and MEP

- 6-minute walking test (6MWT)

- dyspnea evaluation (Borg scale)

- systemic inflammation evaluation by measuring the hypersensitive fraction of C-reactive protein (hsCRP).

A spirometer (Jaeger, Wuerzburg, Germany) with shutter module for analysis of muscular respiratory pressures was used. The obtained values were expressed as percentages from ideal values. The force of respiratory muscles may be evaluated using the static measurements (MIP, MEP), or it may result from dynamic maneuvers (MVV - voluntary maximal ventilation).

MIP represents the highest subatmospheric pressure that can be generated during an inspiration against a blocked airway (Muller maneuver). MEP is the highest pressure that can be achieved during a high expiratory effort against a blocked airway (Valsalva maneuver). The method is usually used by starting the maneuver from the residual volume for MIP determination and from the maximal capacity for MEP determination. There are just a few contraindications for these exploratory maneuvers: aneurysm, uncontrolled hypertension, urinary infection, recent abdominal or thoracic surgery.

The subjects underwent between three and five maximal acceptable and reproducible maneuvers (with differences of 
$3 \%-9 \%$ between values). For the statistical evaluation, the maximal value obtained from these successive trials was taken into consideration. An approximate one minute interval was permitted between consecutive efforts.

The technique used followed the principles of Black and Hyatt. A minimal leak of air (shutter module) should be used in order to prevent the blocking of the epiglottis. This minimal leak has no influence on recording measurements. The inspiratory or expiratory effort has to be sustained for a minimum of one second.

The 6MWT was performed according to the American Thoracic Society and the European Respiratory Society (ATS/ERS) standard on a 30-m long and 1.5-m wide, flat corridor inside the hospital. The patients were asked to walk the maximal distance they could for 6 minutes. They were monitored by a doctor who assisted them, periodically encouraged them, and informed them about the remaining time and the possibility that some adverse clinical signs might appear. $\mathrm{O}_{2}$ saturation was assessed using a Nonin (Onys, USA) pulse oximeter and was recorded at 3 minutes at the beginning of the testing and at 6 minutes at the end of the testing. Cardiac parameters (blood pressure $[\mathrm{BP}]$ and heart rate $[\mathrm{HR}]$ ) were continuously monitored during the test and the dyspnea score on the Borg scale was also assessed. The test was performed twice at an interval of 60 minutes and we chose the best value.

Hand muscle strength was recorded with a dynamometer (Dynatest, Jungingen, Germany). The record was achieved after the following procedure: the subject takes the dynamometer in his hand and squeezes it as hard as possible, without further movement. Two recordings are required and only the highest values obtained on the pressure scale of the dynamometer measured in kilograms force were taken into consideration. The procedure was performed with the dominant hand. Obtained values are expressed in $\mathrm{kgF}$.
In order to determine body composition a $310 \mathrm{e}$ Biodynamics impedance meter was used (Body Composition Analyzer, USA), that is able to provide data concerning: lean body weight (LBW; total weight without fat); fat body weight (FBW, mass of body fat); and percentage of body fat $(\% \mathrm{BF})$. Although FBW and \%BF may vary (heart disease, salt diet, etc), LBW represents a more stable and specific parameter than BMI or anthropometric parameters in assessing muscle mass.

hsCRP is a parameter that reflects systemic inflammation, normal values being $\leq 6 \mathrm{IU} / \mathrm{L} .{ }^{13}$

Statistical analysis and graphic representation of the data were performed using the software programs Microsoft Excel 2003 (Microsoft Corporation, Redmond, WA, USA) and GraphPad Prism 4 (GraphPad Software, La Jolla, CA, USA). All presented data are expressed as mean \pm standard deviation.

Statistical comparison of the data from all groups was performed by means of analysis of variance tests (ANOVA) followed by post-hoc analysis using the Dunnett multiple comparisons test. The power link between certain studied variables was assessed using the Pearson's correlation coefficient. Statistical significance was considered when $P<0.05$. We used the scale of assessment by Beaglehole (1997), regardless of the purpose of the association: strong $(\mathrm{r}>0.70$ ), moderate ( $\mathrm{r}$ between $0.40-0.70$ ), weak ( $\mathrm{r}$ between $0.20-0.40)$, and absent $(\mathrm{r}<0.20$.)

\section{Results}

The results of the investigations according to each stage of COPD, with the significant differences compared to the control group, are presented in Table 2.

MIP $(\mathrm{kPa})$ was found to be significantly decreased in mild-severe stages of COPD. Muscle force (kgf) assessed by dynamometry was found to be decreased only in advanced

Table 2 The relationship between different variables and the severity of COPD

\begin{tabular}{|c|c|c|c|c|c|c|}
\hline & $\begin{array}{l}\text { Control } 3 \text { I } \\
\text { patients }\end{array}$ & $\begin{array}{l}\text { COPD I } 4 \\
\text { patients }\end{array}$ & $\begin{array}{l}\text { COPD II } 34 \\
\text { patients }\end{array}$ & $\begin{array}{l}\text { COPD III } 33 \\
\text { patients }\end{array}$ & $\begin{array}{l}\text { COPD IV } 50 \\
\text { patients }\end{array}$ & \\
\hline MIP (kPa) & $7.9 \pm 2.6$ & $7.74 \pm 3.99$ & $6.19 \pm 2.42 *$ & $5.35 \pm 2.49 * * *$ & $4.56 \pm 1.98 * * *$ & $P<0.001$ \\
\hline MEP (kPa) & $10.84 \pm 2.76$ & $10.07 \pm 3.6$ & $8.64 \pm 2.88^{*}$ & $8.32 \pm 3.19 * *$ & $7.9 \pm 3.14 * * *$ & $P<0.01$ \\
\hline Dynamometry (kgF) & $0.71 \pm 0.16$ & $0.55 \pm 0.13$ & $0.53 \pm 0.15^{* * *}$ & $0.47 \pm 0.12^{* * *}$ & $0.41 \pm 0.07 * * *$ & $P<0.05$ \\
\hline LBW (kg) & $66.2 \pm 12$ & $58.6 \pm 10.11$ & $57.16 \pm 9.69 * *$ & $56.89 \pm 13.11 * *$ & $54.49 \pm 9.79 * * *$ & $P<0.001$ \\
\hline 6MWT (m) & $544 \pm 87$ & $515 \pm 59$ & $472 \pm 82 *$ & $350 \pm 99 * * *$ & $84 \pm 129 * * *$ & $P<0.001$ \\
\hline $\mathrm{SaO}_{2}(\%)$ & $97 \pm 2$ & $97 \pm 1$ & $96 \pm 3$ & $94 \pm 2^{* *}$ & $89 \pm 5 * * *$ & $P<0.001$ \\
\hline $\mathrm{FEV}_{1}(\mathrm{~L} / \mathrm{s})$ & $2.90 \pm 0.25^{* *}$ & $2.49 \pm 0.24$ & $1.93 \pm 0.27 * * *$ & $1.15 \pm 0.17^{* * * *}$ & $0.74 \pm 0.26 * * *$ & $P=0.036$ \\
\hline $\mathrm{FEV}_{1}$ (\% pred) & $96.76 \pm 8.66^{*}$ & $83.6 \pm 8.39$ & $64.34 \pm 9.21 * * *$ & $38.65 \pm 5.95^{* * *}$ & $24.84 \pm 8.98 * * *$ & \\
\hline
\end{tabular}

Note: $* P<0.05$ vs control, $* * P<0.01$ vs control, $* * * P<0.001$ vs control.

Abbreviations: MIP, maximal inspiratory pressure; MEP, maximal expiratory pressure; LBW, lean body weight; 6 MWT, 6 minutes walking time; FEV ${ }_{1}$, forced expiratory volume in one second. 
stages of the disease. MIP values were well correlated (r $=0.53, P=0.0003$ ) with $6 \mathrm{MWT}$ (Figure 1). This final test was also found to be correlated $(\mathrm{r}=-0.68, P=0.0004)$ with CRPhs values. No significant correlation could be noticed in the case of the relation between MIP and CRPhs.

We also found a mild decreased association between forced expiratory volume in one second $\left(\mathrm{FEV}_{1}\right)$ and $6 \mathrm{MWT}$.

\section{Discussion}

Hospitalizations caused by E-COPD are responsible for a state of prolonged physical inactivity due to a variable degree of muscle wasting. Decreased muscle strength induced by E-COPD is due to metabolic and nutritional dysfunctions, oxidative stress, and an amplification of the inflammatory status. Bed rest and systemic glucocorticoid treatment also contribute to decreased muscle strength.

This explains that in the 6MWT test only $35 \%$ of the patients with stage III E-COPD had finished the test and only $11 \%$ of the patients with stage IV E-COPD have performed this test, showing a weak to moderate association between MIP and 6MWT in these stages.

Information obtained after testing the peripheral and respiratory muscles may be helpful in establishing the type of physical training. Free muscle mass (LBW) is inversely correlated with disease severity: stage IV patients obtained the lowest values $(P<0.001)$, which means a very reduce muscular mass.

Pulmonary function (as reflected by $\mathrm{FEV}_{1}$ ) diminishes once with the beginning of E-COPD and is recovering slowly ( $\cong 8.6 \%) 2$ months later, one of the factors responsible for monitoring is very likely $\operatorname{MIP}(\Delta=1.65 \mathrm{kPa}$, $P=0.003)$.

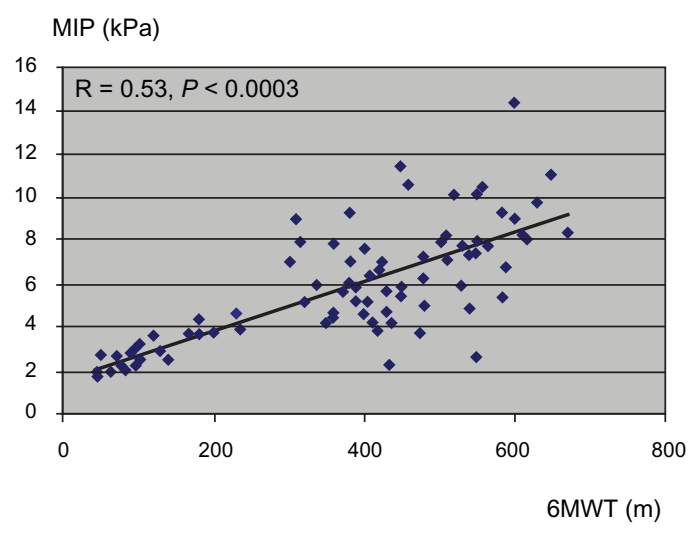

\section{Correlation between MIP and 6MWT}

A moderate degree of systemic inflammation is today considered a primary factor in the development of COPD, cachexia/muscle wasting, and cardiovascular diseases. hsCRP, as an inflammatory marker, was found to have increased in the stable phase of COPD and even more during exacerbation.

The difference between acute and stable phases is not significant $(P=0.41)$ because many patients were in an advanced stage of disease and $40 \%$ of them had associated cardiovascular diseases.

No significant correlations between MIP and hsCRP were found. This could be due to the fact that hsCRP is a global marker of inflammation, not as specific for muscle wasting as tumor necrosis factor or interleukin-6.

\section{Study limitations}

Comparison of parameters in the same patient was not performed in acute or stable phases, although the groups could be compared in terms of disease stage, age, sex, tobacco use status, etc. There was a numerical difference between groups (E-COPD and control group). It was difficult to include more patients in the control group with similar demographic data to those included in the study group (healthy patients with no other pathologies that influence the accuracy of the control group).

\section{Contributions of our study}

Correlation of MIP with other parameters (respiratory, muscular, inflammatory) in E-COPD and stable COPD.

\section{Conclusions}

Respiratory muscles fatigue is increased during E-COPD and a reverse correlation between MIP and the severity of COPD

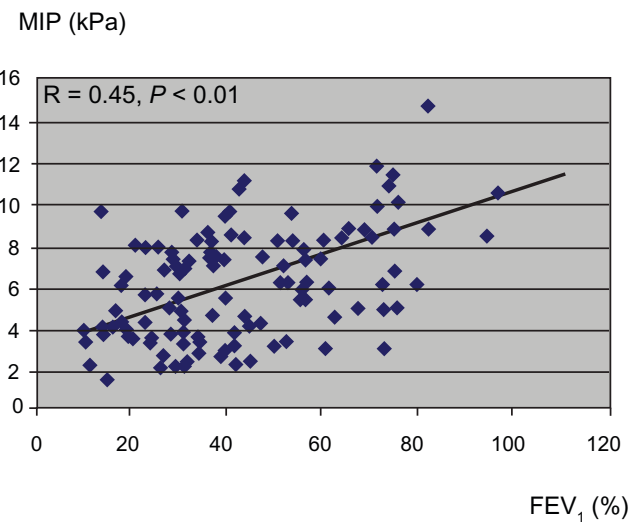

Correlation between MIP and FEV $_{1}(\%)$

Figure I Different correlation between MIP and 6MWT and between MIP and FEV,

Abbreviations: $\mathrm{FEV}_{1}$, forced expiratory volume in one second; MIP, maximal inspiratory pressure; 6MWT, 6-minute walk test. 
was observed, as well as a direct correlation between MIP and $\mathrm{FEV}_{1}$. There is a significant correlation between MIP and effort capacity (6MWT) similar to the relation between peripheral muscles forces and the 6MWT.

Together with other markers, MIP and MEP represent markers for assessing the degree of respiratory muscle dysfunction. These parameters can be useful for the diagnosis, follow up, and prognosis of patients included in respiratory rehabilitation programs, but also in other respiratory or muscle pathologies or in healthy subjects (such as athletes).

\section{Disclosures}

The authors report no conflicts of interest in this work.

\section{References}

1. American Thoracic Society and the European Respiratory Society. Skeletal muscle dysfunction in chronic obstructive pulmonary disease. Am J Respir Crit Care Med. 1999;159:S1-S40.

2. Tudorache V, Mihăicuță Șt, Csunderlick H, Disfuncția mușchilor ventilatori scheletici - o revizie a patogeniei BPOC. Pneumologia. 2001;1:33-38

3. Gosselink R, Troosters T, Decramer M. Distribution of respiratory and peripheral muscle weakness in patient with stable COPD. J Cardiopulm Rehabil. 2000;20:353-358.
4. Troosters T, Pitta F, Decramer M. Respiratory muscle assessment in pulmonary rehabilitation. In: Claudio F. Donner, editor. Pulmonary Rehabilitation. London: Hodder Arnold; 2005; p. 69-79.

5. Orozco-Levi M. Structure and function of the respiratory muscles in patients with COPD: impairment or adaptation? Eur Respir J. 2003;:Suppl 46:S41-S51.

6. Celli BR, Cote CG, Marin JM, et al. The body-mass index, airflow obstruction, dyspnea and exercise capacity index in chronic obstructive pulmonary disease. N Engl J Med. 2004;350:1005-1012.

7. Sarmiento AR, et al. Inspiratory muscle training in patients with chronic obstructive pulmonary disease. Am J Respir Crit Care Med. 2002;1666:1491-1497.

8. Spruit MA, et al. Muscle force during an acute exacerbation in hospitalised patients with COPD and its relationship with CXCL8 and IGF-I. Thorax. 2003;58:752-756.

9. Gayan-Ramirez G, Decramer M. Miopathie peripherique de BPCO. Rev Med Suisse. 2002;614.

10. Perez T. Comment explorer en premiere intention les muscles respiratoires? Rev Mal Respir. 2005;22:2S37-2S46.

11. Tudorache $\mathrm{V}$, et al. How relevant is maximal inspiratory pressure in the evaluation of muscle disfunction in COPD. Eur Respir J. 2008; 32 Suppl 2:52.

12. Global Initiative for Chronic Obstructive Lung Disease (GOLD). Global Strategy for the Diagnosis, Management and Prevention of Chronic Obstructive Pulmonary Disease. 2006.

13. Anderson GP. COPD, asthma and C-reactive protein. Eur Respir J. 2006;27:874-876.
International Journal of COPD

\section{Publish your work in this journal}

The International Journal of COPD is an international, peer-reviewed journal of therapeutics and pharmacology focusing on concise rapid reporting of clinical studies and reviews in COPD. Special focus is given to the pathophysiological processes underlying the disease, intervention programs, patient focused education, and self management protocols.

\section{Dovepress}

This journal is indexed on PubMed Central, MedLine and CAS. The manuscript management system is completely online and includes a very quick and fair peer-review system, which is all easy to use. Visit http://www.dovepress.com/testimonials.php to read real quotes from published authors. 